\title{
Efecto placebo y estimulación sonora binaural de ondas beta y theta en el rendimiento de una tarea de memoria de trabajo
}

\section{Placebo Effect and Binaural Sound Stimulation of Beta Wave and Theta Wave in a Working Memory Task Performance}

\author{
Sebastián Alonso Rojas-Álvarez $\underline{\mathrm{ORCID}}$, Ancizar Valencia ${ }^{2} \underline{\mathrm{ORCID}}$, Mauricio Alberto Barrera- \\ Valencia ${ }^{3} \underline{\mathrm{ORCID}}$ \\ 123 Universidad de Antioquia \\ 2 Universidad Nacional \\ ${ }^{3}$ Hradec Králové University \\ Colombia
}

Fecha correspondencia:

Recibido: enero 21 de 2019.

Aceptado: septiembre 5 de 2019.

Forma de citar:

Rojas-Álvarez, S., Valencia, A. \& Barrera-Valencia, M. (2020). Efecto placebo y estimulación sonora binaural de ondas beta y theta en el rendimiento de una tarea de memoria de trabajo. Rev.CES Psico, 13(1), 32-51.

Open access

(c) Copyright

Licencia creative commons

Etica de publicaciones

Revisión por pares

Gestión por Open Journal System DOl: http://dx.doi.org/10.21615/ cesp.13.1.3

ISSN: 2011-3080

\section{Resumen}

En el presente trabajo se realizó un estudio cuasi experimental de medidas repetidas con grupos caso y control, cuyo objetivo fue medir el efecto de los sonidos binaurales de onda beta y theta, y de la información placebo relacionada a ellos, sobre el rendimiento de una tarea de memoria de trabajo computarizada, versión libre del Automated Operation Span Task-AOSPANde Unsworth, Heitz, Schrock y Engle (2005). Dicha tarea se repitió en dos sesiones separadas por un lapso mínimo de dos semanas, una de las cuales se resolvía mientras se escuchaba una estimulación sonora binaural de onda beta o theta que, dependiendo del grupo, se acompañaba o no de información placebo. Se evaluaron 98 personas con distinto nivel de escolaridad divididas en seis grupos experimentales y uno de control. Se encontraron diferencias significativas en el tiempo de presentación de la tarea experimental asociada a información placebo, mostrando una mejoría en la segunda aplicación de la tarea. Debido a ambigüedades en los resultados, a pesar de encontrar diferencias significativas asociadas a la estimulación binaural de onda beta, no pudieron establecerse relaciones concretas entre la mejoría en el recuerdo de conjuntos de hasta cuatro elementos con la presencia de dicha variable. No se encontraron diferencias significativas en las variables de recuerdo global, lo cual puede explicarse por el tipo de tarea de memoria utilizado.

Palabras clave: Memoria de trabajo, Sonidos binaurales, AOSPAN, Evaluación computarizada, Psychopy, Procesamiento de la información, recuerdo inmediato, Psicología Experimental, Estimulación sonora, Memoria a corto plazo, Pruebas de interferencia.

\section{Abstract}

In this work, a quasi-experimental study of repeated measures with case and control groups was carried out, whose objective was to measure the effect of binaural sounds of beta and theta waves, and the placebo informa- 


\section{Sobre los autores:}

1. Psicólogo. Psicoeducador en Centro Transitorio de Menores infractores y en la Secretaría de Educación del municipio de Santa Rosa de Osos (Antioquia, Colombia)

2. Ingeniero de Sistemas y Computación. Estudiante de Maestría en Psicología de la Universidad de Antioquia. Investigador del Grupo de Investigación y Desarrollo en Inteligencia Artificial - GIDIA-, Universidad de Antioquia.

3. Doctor en Psicología con Orientación en Neurociencias Cognitivas. Magister en Neuropsicología. Psicólogo. Investigador Asociado del Grupo de investigación Psicología Cognitiva, Jefe de Movilidad y Relaciones Internacionales, Facultad de Ciencias Sociales y Humanas, Universidad de Antioquia. Docente invitado de la Universidad Hradec Kralové, República Checa. tion related to them, on the performance of a computerized working memory task, Automated Operation Span Task -AOSPAN- free version of Unsworth, Heitz, Schrock and Engle (2005). This task was repeated in two sessions separated by an interval of two weeks, one of which was resolved while listening to a binaural sound stimulation of beta wave or theta that, depending on the group, was supported or not by placebo information. 98 people with different levels of schooling were tested twice, divided into six experimental groups and one control group. Significant differences were found related to time duration in performing the experimental task associated with placebo information; it was showed an improvement the second time the task was administered. Due to ambiguities in the results, despite finding significant differences associated with binaural stimulation of beta wave, it was not possible to establish concrete relations between the improvement in the memory of sets up four elements with the presence of this variable. No significant differences were found in the global recall variables, which can be explained by the type of memory task performed.

Keywords: Working Memory, Binaural Sounds, AOSPAN, Computerized Assessment, Psychopy, Information Processing, Immediate Memory, Psychology, Experimental Psychology, Sound Stimulation, Short-Term Memory, Interference Tests.

\section{Introducción}

La memoria de trabajo u operativa es aquella porción de la memoria que permite actuar sobre la información nueva de manera que pueda retenerse temporalmente y manipularse en función de la realización de cualquier tarea cognitiva que requiera la integración de dicha información con porciones mnésicas ya almacenadas (Atkinson \& Shiffrin, 1971; Baddeley \& Hitch, 1974; D’Esposito \& Postle, 2015; Solís \& López-Hernández. 2009). Este sistema consta de tres componentes primordiales: un ejecutivo central, un lazo fonológico y una agenda visoespacial (Aguilar, 2004; Etchepareborda \& Abad-Mas, 2005; Stelzer, Andés, Canet-Juric, \& Introzzi, 2016), convirtiendo a la memoria de trabajo en un mecanismo operativo cuyo componente nuclear, el ejecutivo central, resulta limitado en cuanto a capacidad y velocidad de almacenamiento, procesamiento y recuperación de la información (Gutiérrez, García, Carriedo, Villa, \& Luzón, 2005). Esto conduce a una competencia por los recursos atencionales del componente ejecutivo central entre el almacenamiento y el procesamiento de la información, que depende de la identificación eficaz de las exigencias del medio y la elaboración de tareas que cumplan con éstas; por tanto, entre más eficaz resulte el proceso, menos recursos consume y habrá una mayor capacidad de almacenamiento (Stelzer et al., 2016).

Todo procesamiento cognitivo implica la activación sincrónica de grupos neuronales capaces de reproducir de forma repetida los patrones de actividad sináptica generados durante la experiencia (Castillo, García, \& Castillo, 2014; Córdoba, Albert, \& López, 2010, Fajardo \& Guzmán, 2016; Leff et al., 2004; Morgado, 2005); estas sinapsis constituyen el sustrato electrofisiológico del procesamiento de la información, y de su plasticidad depende la eficacia del mismo (Clemenson, Gage, \& Stark, 2018, Ortega Loubon \& Franco, 2010).

Dicha plasticidad puede inducirse, ya sea mediante la aplicación de trenes de estimulación de alta frecuencia que exciten la actividad conjunta de pares sinápticos asociados, de tal forma que aferencias fuertes provoquen un incremento en la proporción de Potenciales a Largo Plazo (PLP), permitiendo un aumento ininterrumpido de la comunicación sináptica que se da gracias a la repetición constante de la activación de una vía (Carasatorre, Ramírez, \& Díaz, 2016; Goto, Yang, \& Otani, 2011; Nicoll, 2017), o por 
Pág 34

Múltiples resultados se han encontrado con respecto al efecto de los distintos rangos de frecuencia binaural en la conectividad cortical y los procesos cognitivos, por los cuales no ha sido posible llegar a un consenso sobre el efecto de esta estimulación en el funcionamiento cognitivo, particularmente en el ámbito de la memoria. medio del fenómeno de sincronización al momento en que la activación presináptica se presenta de forma simultánea con la despolarización de la membrana postsináptica (Balderas, Ramírez, \& Bermúdez, 2004; Dodla \& Wilson, 2017). Con base en esto se han buscado formas de estimulación que aumenten la plasticidad (Castillo et al., 2014; Figueiras et al., 2009; Freigni \& Pascual-Leone, 2007; Pluta, 2011), entre las cuales se encuentran los sonidos binaurales (Beauchene et al., 2016; Gonzales, 2013).

Los sonidos binaurales se han propuesto como un tipo de estimulación no invasiva enfocada en la generación de potenciales evocados de carácter auditivo que impulsan la sincronización neuronal mediante la exposición a dos sonidos de distinta frecuencia que, escuchándose al mismo tiempo, pueden generar un pulso de frecuencia equivalente a la diferencia entre las dos frecuencias iniciales; provocando lo que en la literatura se ha denominado respuesta de seguimiento de frecuencia (FFR, por sus siglas en inglés), la cual consiste en la sincronización de áreas cerebrales específicas que se da como resultado de un seguimiento de la actividad ondulatoria del córtex frente a la frecuencia presentada por el pulso antes mencionado (Becher, et al., 2014; Chaieb et al., 2017; Gao, et al., 2014; Llancafil, 2013; Vernon, Peryer, Louch, \& Shaw, 2012).

Múltiples resultados se han encontrado con respecto al efecto de los distintos rangos de frecuencia binaural en la conectividad cortical y los procesos cognitivos, por los cuales no ha sido posible llegar a un consenso sobre el efecto de esta estimulación en el funcionamiento cognitivo, particularmente en el ámbito de la memoria. Con respecto al efecto de los sonidos binaurales sobre los procesos mnésicos, se ha encontrado una predominancia en los estudios del efecto de bandas binaurales de onda theta (entre 3.5 y 7.5 Hz) (Chaieb, et al., 2017; Gonzales, 2013; Ledesma \& Sanchez, 2016; Seifi Ala, Ahmadi-Pajouh, \& Nasrabadi, 2017; Wahbeh, Calabrese, Zwickey, \& Zajdel, 2007) y beta (Entre $12 \mathrm{~Hz}$ y $30 \mathrm{~Hz}$ ) (Beauchene et al., 2016; Gao et al., 2014; Kennerly, 1994; Vernon et al., 2014), con una variación en los resultados que puede deberse a la metodología empleada en cada uno de los estudios.

Si bien se parte del supuesto de que hay un efecto de los sonidos binaurales sobre la cognición, no se puede dejar de lado la problemática del control de expectativas sobre los grupos control en los estudios psicológicos (Boot, Simons, Stothart, \& Strutts, 2013), dejando aún vigente el efecto del placebo sobre los mecanismos dopaminérgicos, de recompensa y el rendimiento cognitivo (Benedetti, Mayberg, Wager, Stohler, \& Zubieta, 2005; de la Fuente-Fernandez et al. 2001; Zatorre, 2015). De hecho, en los estudios mencionados anteriormente no se especifica la presentación del estímulo ante los grupos participantes, lo cual deja abierta la incógnita de dicho efecto sobre el rendimiento de las tareas evaluadas en cada uno de ellos.

Debido a lo anterior, el presente estudio se propone medir el efecto de los sonidos binaurales de onda beta y theta, con y sin información placebo, sobre el rendimiento de una tarea de memoria de trabajo computarizada, para establecer tanto el efecto de dichas variables sobre el procesamiento ejecutivo como el posible efecto de la expectativa de rendimiento sobre el rendimiento mismo de esta tarea.

\section{Método}

Estudio cuasi experimental de medidas repetidas con grupos caso y control, en el que se creó una tarea computarizada de interferencia en la que los participantes debían recordar una serie de letras a medida que realizaban operaciones matemáticas simples. Dicha tarea se repitió en dos sesiones separadas por un lapso mínimo de dos semanas, 
una de las cuales se resolvía mientras se escuchaba una estimulación sonora binaural de onda beta o theta que, dependiendo del grupo, se acompañaba o no de información placebo.

\section{Tarea Experimental}

Se desarrolló una versión libre y modificada del Automated Operation Span Task (AOSPAN) de Unsworth, Heitz, Schrock y Engle (2005), versión alternativa computarizada del Operation Span Task creada por Turner y Engle (1989), que representa una prueba de amplitud compleja de memoria de trabajo e implica tanto una tarea de recuerdo (tarea primaria) como un componente de razonamiento/procesamiento (tarea secundaria o de interferencia).

La tarea primaria, en este caso, es una de retención de letras en orden directo, en la que, antes de la presentación de cada letra se debe resolver un problema matemático simple (Interferencia); se denomina set a un conjunto que puede tener un tamaño de tres, cuatro, cinco o seis letras; de cada set, se presenta primero una letra y luego una operación matemática simple que hace las veces de tarea de interferencia, repitiendo esto hasta que cada letra del set se haya mostrado (Figura 1). Cada set tiene tres conjuntos de letras distintos, así habrá tres conjuntos de letras para el set de tres, tres para el de cuatro y así sucesivamente. Para el desarrollo de esta tarea se recurrió al software de programación libre Psychopy (Pierce, 2007), en su versión 1.90.2. Bajo los estándares del programa original (Unsworth et al., 2005), se desarrolló la misma tarea con las siguientes modificaciones:

Interfaz

Se realizó una traducción de las cadenas de texto al español; se hizo una aleatorización de la presentación de ecuaciones matemáticas simples; se implementó el teclado como entrada de respuesta, dejando de lado el uso del cursor como se hacía en la prueba original; se suprimió el tablero de recuperación que mostraba las letras en pantalla, dejando disponible únicamente un espacio para la digitación de las letras recordadas; se hizo una inversión en el orden de presentación de los pares de estímulos, cuyo orden original era una ecuación seguida de una letra a recordar, cambiándolo por la presentación de una letra a recordar, seguida de una ecuación, esto con el fin de que la primera ecuación de cada set siga funcionando como tarea de interferencia ante el recuerdo de la primera letra (Figura 1); se decidió reducir, de $85 \%$ a $80 \%$, el porcentaje mínimo de ecuaciones correctas solicitado durante la prueba, puesto que se evidenció, en la prueba piloto, que un número elevado de sujetos de la muestra no llegaba al primer porcentaje; se realizó un cambio en los sets presentados en la fase de prueba y familiarización de la tarea, pasando de la presentación original de tres sets de dos pares de elementos, a la presentación de un set de tres y un set de cuatro, con el propósito de propiciar un mejor acercamiento a la tarea experimental; y se disminuyó el tamaño de los sets presentados en la tarea experimental, pasando de cinco sets (de tres a siete letras) a cuatro sets (de tres a seis letras), cada uno con tres repeticiones.

\section{Puntajes}




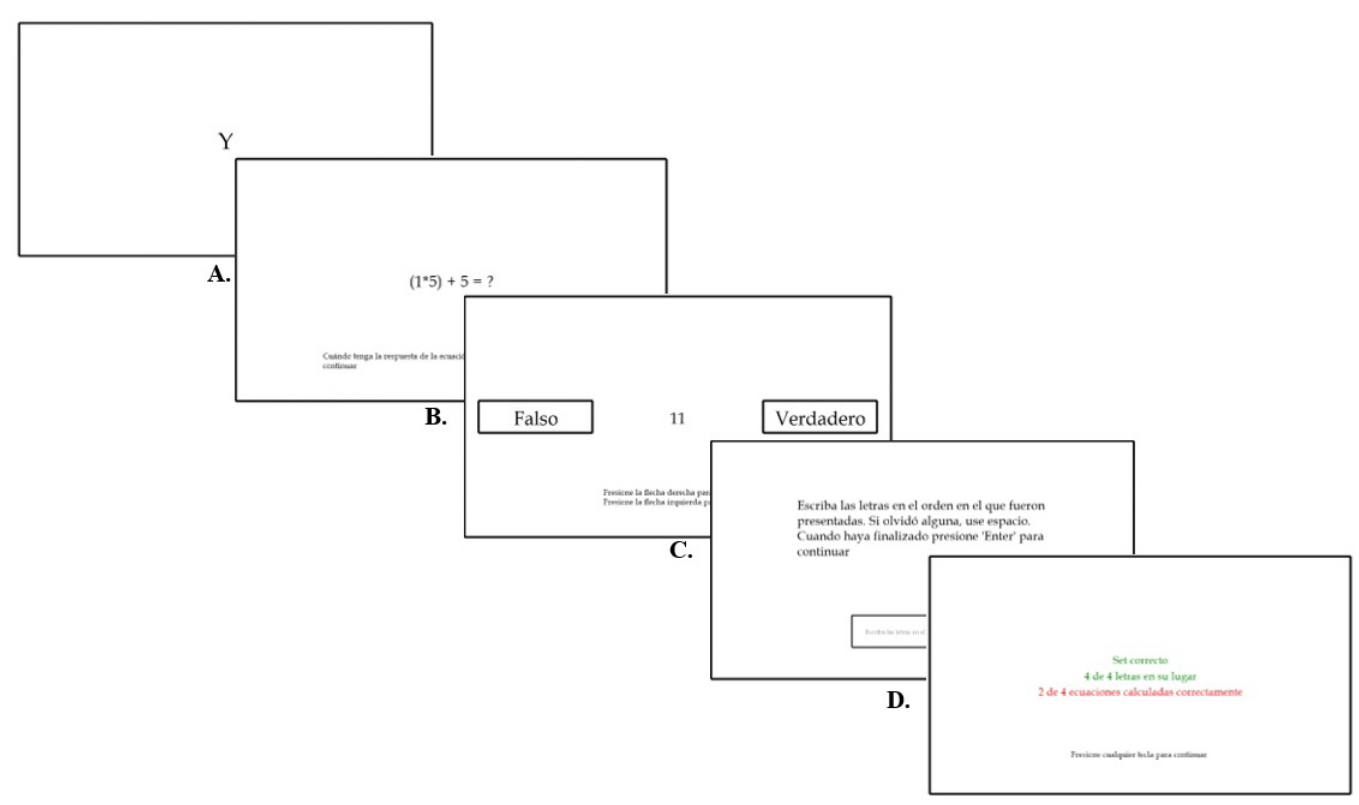

E.

Figura 1. Flujo de tarea experimental. A. Se presenta la letra que hace parte del set que se debe recordar. B. Se presenta una operación matemática aleatoria, que se queda en pantalla por un tiempo dinámico que depende del tiempo que le tomó al participante resolver quince operaciones matemáticas aleatorias. C. El participante debe usar las flechas del teclado (derecha para sí, izquierda para no) para responder si el resultado que se le muestra es la respuesta a la ecuación del punto B. Si el participante se consume el tiempo promedio por ecuación y no proporciona una respuesta, el software tomará la respuesta como equivocada y avanzará a la siguiente pantalla. Los pasos A, B y C, se repiten hasta que todas las letras del set hayan sido mostradas. D. Se muestra una pantalla de recuperación en la que el participante debe introducir, en el orden en el que se le presentaron, las letras del set. E. Cada vez que se termina un set, se le presenta al participante una ventana en la que se le muestra su desempeño en dicho set.

El AOSPAN original, ejecutado mediante el Software Inquisit 5, permite adquirir datos sobre la velocidad de respuesta dada por el sujeto durante la prueba y cinco puntajes específicos: OSPAN total, Letras recordadas, Ecuaciones resueltas incorrectamente, Ecuaciones fallidas por tiempo y Total de ecuaciones fallidas (Unsworth et al., 2005). Estos valores se conservaron sin modificaciones en la nueva versión, y se adicionaron los siguientes puntajes nuevos:

1. Sets completos: Número de sets recordados en su totalidad y en orden correcto; este puntaje se divide en cuatro subpuntajes que permiten ver específicamente cuantos sets de cada tamaño se recordaron de manera correcta en cada una de las tres presentaciones por set (sets de tres, sets de cuatro, sets de cinco y sets de seis letras).

2. Tiempo total de la fase de prueba - medido en minutos (TTFPm): Se refiere al tiempo que le tomó al participante resolver la tarea experimental, que consta de 12 sets de letras y 54 operaciones matemáticas.

3. Tiempo promedio para cada ecuación - medido en segundos (TPPCEs): Corresponde al tiempo promedio obtenido en la solución de ecuaciones en el periodo de familiarización matemática. Este puntaje permite ver cuál fue el techo límite en la velocidad de respuesta matemática en la tarea experimental por individuo.

4. Sets con letras adicionadas: Marca el número de sets en los que el participante 
acertó, pero agregó una letra de más.

5. Sets completos en desorden: Muestra el número de sets en los que el participante recordó las letras correctas, pero no su orden.

6. Sets correctos con espacios: Presenta el número de sets en los que el participante agregó un espacio en la posición de una letra olvidada mientras el resto de las letras están posicionadas de forma correcta.

\section{Confiabilidad}

Para evaluar la confiabilidad de la tarea se tomaron los resultados de 33 personas que pasaron el umbral de interferencia del $80 \%$, y se evaluó el alfa de Cronbach con las 10 variables anteriormente establecidas, dando como resultado un alfa de 0,715, valor que aumenta a 0,758 al eliminarse el ítem de ecuaciones fallidas por cálculo; adicionalmente, se tomaron los datos de los individuos que pasaron el umbral de interferencia del 70\%, dejando un $n=41$ y un alfa de 0,704, que se elevaba a 0,761 al eliminarse, de nuevo, el ítem ecuaciones fallidas por cálculo. Se decidió conservar, aun así, esta variable dentro del programa, ya que, junto al ítem ecuaciones fallidas por tiempo, permite contrastar con mayor facilidad los resultados del rendimiento matemático, descartando con esto cualquier error en la ventana de salida del programa; sin embargo, no se tomó como variable de evaluación dentro del análisis de la tarea experimental debido a su nivel de interferencia en la fiabilidad de la prueba.

\section{Estímulos sonoros}

Para la creación de los estímulos sonoros utilizados, se usó una misma pista de música clásica, que se modificó con el programa Cool Edit pro, programa de edición avanzada de sonido en el que se tomó una pista en formato wav y se transformó a binaural en un rango de onda beta $(17 \mathrm{~Hz})$ y en un rango de onda theta $(7 \mathrm{~Hz})$.

Para la aplicación de información placebo se optó por una presentación de 5 a 10 minutos en Power point en la que se siguió la siguiente matriz lógica: Definición de sonidos binaurales - Efectos de sonidos binaurales en el procesamiento cognitivo Definición de sincronización neuronal - Ejemplificación de sincronización Neuronal (Castillo, García, \& Castillo, 2014) - Usos actuales (Presentación del instituto Monroe - Audio Aspirina de Bayern) - Bibliografía. Esta matriz de presentación se utilizó para mostrar la supuesta eficacia de los sonidos binaurales respaldados por investigaciones científicas, instituciones americanas y productos comerciales; luego de la presentación se les indicó a los participantes que la exposición a los sonidos binaurales haría que su rendimiento en la tarea mejorara de forma considerable y que la finalidad del estudio era medir dicha mejora.

\section{Participantes}

Para la aplicación de la tarea se formaron seis grupos experimentales y un grupo control. Los grupos fueron compuestos por un total de 98 participantes voluntarios, 47 hombres, 51 mujeres, todos con educación media o superior, con edades que oscilaban entre los 15 y los 34 años, seleccionados y categorizados de forma aleatoria. Se realizó una encuesta demográfica previa a la prueba con el fin de confirmar que los participantes no presentaran dificultades auditivas o se encontraran bajo el efecto de sustancias psicoactivas. Todos los participantes firmaron un consentimiento informado que leyeron antes de la aplicación de la prueba, teniendo la oportunidad de resolver todas las dudas pertinentes y con el conocimiento de que podían cancelar la prueba en el momento en que quisieran; además, en este con- 
sentimiento se explicitó que los datos obtenidos en el estudio se utilizarían en pro de la presente investigación, aclarando la posibilidad de un uso posterior de estos en nuevas investigaciones cuyo objetivo o propósito no se especificaba en el presente formato. Los menores de edad firmaron el respectivo asentimiento y entregaron el consentimiento informado firmado por sus padres o tutores legales. Dentro de los seis grupos experimentales se realizó una división en la presentación de estímulos independientes y una distribución muestral que se exhibe en la Tabla 1.

Variables dependientes (Vd) e independientes (Vi)

Tabla 1. Distribución Muestral de participantes

\begin{tabular}{|c|c|c|c|c|c|c|c|}
\hline Grupo & $n$ & Estimulación & $\begin{array}{c}\text { Orden de } \\
\text { presentación }\end{array}$ & $\begin{array}{l}\text { n por orden de } \\
\text { presentación }\end{array}$ & \multicolumn{2}{|c|}{$\begin{array}{c}\text { n por } \\
\text { sexo }\end{array}$} & $\begin{array}{l}\text { Edad (Mínimo/ } \\
\text { Máximo - Media) }\end{array}$ \\
\hline \multirow{4}{*}{1} & \multirow{4}{*}{16} & \multirow{4}{*}{ MC } & \multirow{2}{*}{1} & \multirow{2}{*}{8} & M & 5 & \multirow{2}{*}{$19 / 26-22.25$} \\
\hline & & & & & $F$ & 3 & \\
\hline & & & \multirow{2}{*}{2} & \multirow{2}{*}{8} & M & 4 & \multirow{2}{*}{$15 / 16-15.50$} \\
\hline & & & & & $\mathrm{F}$ & 4 & \\
\hline \multirow{4}{*}{2} & \multirow{4}{*}{16} & \multirow{4}{*}{ MCP } & \multirow{2}{*}{1} & \multirow{2}{*}{8} & M & 4 & \multirow{2}{*}{$15 / 34-18.88$} \\
\hline & & & & & $\mathrm{F}$ & 4 & \\
\hline & & & \multirow[b]{2}{*}{2} & \multirow{2}{*}{8} & $M$ & 4 & \multirow{2}{*}{$15 / 27-17.63$} \\
\hline & & & & & $\mathrm{F}$ & 4 & \\
\hline \multirow{4}{*}{3} & \multirow{4}{*}{16} & \multirow{4}{*}{ MCB } & \multirow{2}{*}{1} & \multirow{2}{*}{8} & M & 4 & \multirow{2}{*}{$15 / 25-19.50$} \\
\hline & & & & & $\mathrm{F}$ & 4 & \\
\hline & & & \multirow{2}{*}{2} & \multirow{2}{*}{8} & M & 4 & \multirow{2}{*}{$15 / 27-18.00$} \\
\hline & & & & & $\mathrm{F}$ & 4 & \\
\hline \multirow{4}{*}{4} & \multirow{4}{*}{15} & \multirow{4}{*}{ MCBP } & \multirow{2}{*}{1} & 7 & M & 3 & $15 / 23-1757$ \\
\hline & & & & 1 & $\mathrm{~F}$ & 4 & $10 / 20-1 / .31$ \\
\hline & & & 2 & 8 & M & 4 & $15 / 27 \quad 1762$ \\
\hline & & & 2 & 0 & $\mathrm{~F}$ & 4 & $15 / 21-1 / .03$ \\
\hline & & & 1 & t & M & 1 & $15 / 16 \quad 1567$ \\
\hline 5 & 11 & MCT & 1 & 0 & $\mathrm{~F}$ & 5 & $15 / 10-15.6 /$ \\
\hline 5 & 11 & MIC I & 2 & 5 & M & 2 & $15 / 20$ \\
\hline & & & 2 & 5 & $\mathrm{~F}$ & 3 & $15 / \angle 4-20.80$ \\
\hline & & & & & M & 1 & \\
\hline 6 & 12 & $M C-1$ & 1 & 6 & $F$ & 5 & $15 / 18-16.33$ \\
\hline 0 & & & & & M & 1 & $01-50$ \\
\hline & & & 2 & 6 & $\mathrm{~F}$ & 5 & $20 / 23-21.50$ \\
\hline Control & 12 & $N / A_{3}+2$ & $N / \Lambda_{0}$ & $N / \Lambda$ & M & 6 & $10 / 25-2200$ \\
\hline Comitiol & 12 & IV/A & IV/A & IV/A & $\mathrm{F}$ & 6 & $191 \angle 3-\angle 2.00$ \\
\hline
\end{tabular}

Donde:

MC: estimulación sonora con música clásica.

MCP: estimulación sonora con música clásica e información placebo.

MCB: estimulación sonora con música clásica transformada a binaural de onda beta.

MCBP: estimulación sonora con música clásica transformada a binaural de onda beta e información placebo.

MCT: estimulación sonora con música clásica transformada a binaural de onda theta.

MCTP: estimulación sonora con música clásica transformada a binaural de onda theta e información placebo.

N/A: No aplica 
Vd: 11 puntajes de la prueba computarizada utilizada, 5 originales y 6 creados (Ver Puntajes de la Tarea Experimental).

Vi: 6 niveles correspondientes a tres tipos de estimulación auditiva diferentes (MC, MCB y MCT), cada una bajo dos condiciones distintas (con o sin información placebo) (Ver Tabla 1).

\section{Aplicación}

Cada participante realizó la tarea experimental en dos ocasiones separadas por un lapso mínimo de dos semanas, una vez con el estímulo independiente y otra sin él. En cada grupo, la mitad de los participantes presentaron la tarea con estímulo independiente durante la primera aplicación (orden 1) y la otra mitad, lo hizo durante la segunda (orden 2), eliminando de esta manera el efecto reactivo intragrupo.

Para la aplicación de la tarea experimental se separó un espacio de cómputo, todos los ordenadores tenían Windows 10 como sistema operativo y a cada uno de ellos se le instaló la versión 1.90.2 del programa Psychopy, junto con el script de la tarea. Cada participante se sentó frente a un ordenador con el programa ya abierto, listo para correr y con una ventana de Windows con el estímulo sonoro preparado para su reproducción. Se instruyó a los participantes sobre el funcionamiento del programa y se les aclaró que la prueba medía rendimiento individual, y dependiendo de si se requería o no, al inicio de cada sesión se presentaba la información placebo.

La presentación del estímulo sonoro se dio desde el inicio de la tarea experimental hasta el final de la misma, a cada participante se le entregó un par de audífonos estéreo cerrados. Se instruyó a los participantes para reproducir el estímulo sonoro antes de iniciar la prueba, también se les solicitó no poner tildes en los nombres para evitar errores automáticos de interpretación de caracteres; se hizo la recomendación de regular el volumen del estímulo sonoro a gusto de cada participante, siempre y cuando se siguiera escuchando, lo cual se confirmó antes de que dieran inicio a la prueba.

Una vez un participante terminaba, se le solicitaba retirarse de la prueba para evitar distracciones a los otros participantes.

\section{Análisis}

Se dividieron los resultados de cada uno de los seis grupos experimentales en función del orden de estimulación mediante el programa estadístico SPSS 23, en el que inicialmente se realizó un ANOVA de medidas repetidas para 4 de las variables dependientes: "Tiempo total de la fase de prueba" (TTFPm), "Tiempo promedio para cada ecuación" (TPPCEs), "Porcentaje de ecuaciones acertadas" y "Ecuaciones fallidas por tiempo", en función de los resultados entre la primera y segunda aplicación como factor intrasujeto (Sesiones) y en función de la ausencia o presencia de información placebo como factor intersujeto; en caso de no cumplir con normalidad se aplicó la prueba de Friedman.

Estas variables fueron analizadas por separado, y con el $n$ total experimental de cada uno de los subgrupos, al considerarlas variables no dependientes de la tarea de interferencia, contrario a las variables resultado de la fase de recuerdo, cuyo análisis es completamente sensible al nivel de interferencia matemática obtenida por los sujetos en ambas sesiones del estudio.

Una vez obtenidos dichos resultados para cada uno de los órdenes de aplicación, 
se decidió eliminar los datos de aquellos participantes cuyo nivel de interferencia, reflejado en el porcentaje de ecuaciones acertadas entre la primera y la segunda aplicación, tuviera una diferencia mayor al 15\%, independientemente de si alcanzó o no el mínimo de interferencia solicitado en la tarea experimental de $80 \%$, esto debido a que el programa original AOSPAN permite un margen de error del $15 \%$ en las comparaciones entre medias de interferencia.

Así las cosas, se procedió a la eliminación de los resultados de 7 participantes cuya diferencia de interferencia entre las dos sesiones fue mayor al 15\%, tal como se muestra en la Tabla 2.

Una vez efectuada esta corrección, se realizó el mismo procedimiento descrito ante-

Tabla 2. Muestras con diferencias mayores al $15 \%$ en ecuaciones acertadas entre aplicaciones

\begin{tabular}{cccc}
\hline & Grupo / Orden & Puntaje Primera Aplicación & Puntaje Segunda Aplicación \\
\hline 1 & $2 / 1$ & 57.41 & 85.19 \\
2 & $3 / 2$ & 48.15 & 66.67 \\
3 & $4 / 2$ & 48.15 & 64.81 \\
4 & $5 / 1$ & 57.41 & 87.04 \\
5 & $5 / 2$ & 88.89 & 66.67 \\
6 & Control & 85.19 & 50.00 \\
7 & Control & 77.78 & 96.30 \\
\hline
\end{tabular}

riormente para los ítems OSPAN, Letras Recordadas, Sets completos, Sets con letras adicionadas y Sets completos en desorden, en cada uno de los grupos de análisis.

Adicionalmente se realizaron ANOVAs de un factor, pareando los resultados de la primera sesión, por un lado, y de la segunda sesión, por otro, entre los dos órdenes de cada variable independiente; de esta manera se utilizó el orden de presentación de la Vi como variable control para delimitar las diferencias entre la aplicación de la tarea con presencia o ausencia de la Vi en una misma sesión entre dos grupos separados.

\section{Resultados}

A partir del análisis de medidas repetidas con factor intrasujeto se encontraron diferencias significativas en los resultados de "TPPCEs" $(P=0.029)$ y "Sets de 6" $(P=0.025)$ en el grupo control, por lo cual se descarta la influencia de los factores independientes en estas dos variables y en las variables individuales "Sets de 3" "Sets de 4" y "Sets de 5"; esto debido a que el valor observado en el grupo control responde al mayor subnivel de sets completos, por lo que una diferencia significativa en una variable de menor nivel tampoco responderían al efecto de la Vi. Por esta razón únicamente se tuvieron en cuenta diferencias significativas en la variable "Sets Completos", que agrupa todos los subniveles, y los subniveles relacionados a este $\mathrm{p}$ valor.

En la Tabla 3 se presentan las medias y la desviación estándar (DS) de las Vd "TPPCEs", "TTFPm", Porcentaje de Ecuaciones acertadas ("\% E. Acert.") y "OSPAN", las cuales mostraron diferencias significativas en el análisis intrasujeto, con sus correspondientes pares intergrupales. En la Tabla 4 se encuentran las medias y la DS de la Vd "Sets Completos", 
y de los subniveles "Sets de 3", "Sets de 4" y "Sets de 6", los dos primeros relacionados con diferencias significativas en la variable Sets Completos en el grupo 3 de orden 1, y el último mostrando, como ya se anotó, diferencias significativas en el grupo control.

Cabe mencionar que, a pesar de haber presentado significancias en el grupo control,

Tabla 3. Medias y Desviaciones Estándar para variables TPPCEs, TTFPm, \% E. Acert. y OSPAN

\begin{tabular}{ccccccccc}
\hline & \multicolumn{2}{c}{ TPPCEs } & \multicolumn{2}{c}{ TTFPm } & \multicolumn{2}{c}{ \% E. Acert. } & Ospan \\
\hline GRUPO & Sesion 1 & Sesion 2 & Sesion 1 & Sesion 2 & Sesión 1 & Sesión 2 & Sesion 1 & Sesion 2 \\
Control & $13,60(7,78)$ & $9,90(4,19)$ & $10,06(2,88)$ & $9,79(3,93)$ & $87,04(7,90)$ & $86,73(13,74)$ & $26,40(15,40)$ & $33,70(13,54)$ \\
MC 01 & $13,09(5,48)$ & $9,89(3,43)$ & $10,61(2,75)$ & $9,55(3,15)$ & $87,27(7,37)$ & $87,50(6,70)$ & $18,50(11,82)$ & $20,63(14,60)$ \\
MC 0 2 & $22,09(7,42)$ & $11,71(2,68)$ & $14,64(1,54)$ & $11,85(1,96)$ & $75,70(8,43)$ & $74,77(8,39)$ & $14,50(7,84)$ & $9,50(11,16)$ \\
MCP 01 & $19,27(7,43)$ & $11,21(4,72)$ & $14,25(5,63)$ & $11,31(4,12)$ & $82,87(7,72)$ & $78,47(13,60)$ & $18,57(15,13)$ & $17,29(13,76)$ \\
MCP 02 & $14,46(, 38)$ & $9,39(2,15)$ & $10,26(1,87)$ & $9,70(1,88)$ & $82,18(13,89)$ & $76,85(11,59)$ & $18,75(12,51)$ & $17,63(6,61)$ \\
MCB 01 & $18,19(5,19)$ & $11,06(1,60)$ & $11,51(1,94)$ & $9,52(0,95)$ & $83,56(8,31)$ & $84,26(7,28)$ & $19,38(9,43)$ & $24,88(12,19)$ \\
MCB 02 & $11,95(2,51)$ & $11,02(1,99)$ & $10,36(2,07)$ & $9,99(0,97)$ & $75,93(14,68)$ & $78,94(9,39)$ & $14,29(11,18)$ & $17,71(9,57)$ \\
MCBP 01 & $18,10(4,51)$ & $10,07(2,17)$ & $11,21(1,02)$ & $9,49(1,16)$ & $76,46(19,00)$ & $72,75(13,43)$ & $15,86(9,86)$ & $21,57(11,47)$ \\
MCBP 02 & $18,26(6,89)$ & $12,80(3,98)$ & $11,85(2,66)$ & $10,19(2,29)$ & $73,61(16,99)$ & $76,62(13,32)$ & $20,43(12,37)$ & $21,57(11,27)$ \\
MCT 01 & $13,03(2,50)$ & $10,02(1,40)$ & $10,72(1,97)$ & $9,67(1,37)$ & $84,88(8,15)$ & $80,25(12,43)$ & $10,20(13,54)$ & $18,40(14,08)$ \\
MCT 02 & $16,56(7,31)$ & $12,49(2,16)$ & $11,77(11,57)$ & $11,18(1,19)$ & $87,78(5,33)$ & $83,70(10,09)$ & $10,00(8,79)$ & $7,00(5,72)$ \\
MCTP 01 & $13,83(2,98)$ & $10,00(1,64)$ & $10,76(1,02)$ & $9,76(1,11)$ & $81,17(7,35)$ & $76,54(8,90)$ & $13,00(6,60)$ & $14,33(9,54)$ \\
MCTP 02 & $14,31(3,99)$ & $11,31(2,23)$ & $10,79(2,18)$ & $9,39(1,90)$ & $84,57(10,91)$ & $88,27(4,63)$ & $19,83(11,58)$ & $17,67(15,37)$ \\
\hline
\end{tabular}

Media (DS) Variables dependientes por Grupo de análisis

Tabla 4. Medias y Desviaciones Estándar para variables Sets Completos, Sets3, Sets4 y Sets6

\begin{tabular}{ccccccccc}
\hline & \multicolumn{2}{c}{ SetsCompletos } & \multicolumn{2}{c}{ Sets3 } & \multicolumn{2}{c}{ Sets4 } & Sets6 \\
\hline GRUPO & Sesion 1 & Sesion 2 & Sesion 1 & Sesion 2 & Sesion 1 & Sesion 2 & Sesion 1 & Sesion 2 \\
Control & $6,30(3,27)$ & $7,70(3,23)$ & $2,10(0,87)$ & $2,10(1,29)$ & $1,80(1,03)$ & $2,20(1,03)$ & $0,90(1,20)$ & $1,60(0,84)$ \\
MC 01 & $4,50(2,51)$ & $4,75(3,41)$ & $1,63(1,06)$ & $1,25(1,28)$ & $1,38(0,92)$ & $1,50(1,20)$ & $0,63(1,06)$ & $0,87(0,84)$ \\
MC 0 2 & $3,75(1,83)$ & $2,25(2,55)$ & $1,75(0,71)$ & $0,88(1,13)$ & $1,00(1,07)$ & $0,25(4,63)$ & $0,25(4,63)$ & $0,25(4,63)$ \\
MCP 01 & $4,29(3,25)$ & $4,14(2,97)$ & $1,29(0,76)$ & $1,43(1,13)$ & $1,14(1,22)$ & $1,14(1,35)$ & $0,86(1,22)$ & $0,57(1,13)$ \\
MCP 02 & $4,88(2,64)$ & $4,63(1,51)$ & $2,25(1,04)$ & $2,13(0,64)$ & $1,38(0,74)$ & $1,25(1,04)$ & $1,00(1,31)$ & $1,25(0,89)$ \\
MCB 01 & $4,63(2,39)$ & $6,38(2,88)$ & $1,63(1,19)$ & $2,50(0,76)$ & $1,25(0,88)$ & $2,37(1,061)$ & $0,75(0,71)$ & $0,38(0,52)$ \\
MCB 02 & $3,42(2,64)$ & $4,43(2,23)$ & $1,00(0,82)$ & $1,86(1,07)$ & $1,14(1,07)$ & $1,14(0,69)$ & $0,29(0,49)$ & $0,43(0,54)$ \\
MCBP 01 & $3,86(2,34)$ & $5,29(2,56)$ & $1,14(0,90)$ & $1,86(0,69)$ & $1,57(1,13)$ & $1,71(1,25)$ & $0,43(0,54)$ & $0,57(0,79)$ \\
MCBP 02 & $5,14(2,73)$ & $5,00(2,58)$ & $2,00(0,82)$ & $1,57(1,27)$ & $1,71(0,95)$ & $1,14(1,22)$ & $0,43(0,54)$ & $0,86(1,07)$ \\
MCT 01 & $2,60(3,29)$ & $4,40(3,05)$ & $1,20(1,30)$ & $1,80(0,84)$ & $0,60(0,89)$ & $1,00(1,00)$ & $0,20(0,45)$ & $1,00(1,00)$ \\
MCT 02 & $2,75(2,22)$ & $2,00(1,63)$ & $1,50(1,00)$ & $1,00(0,82)$ & $0,75(0,96)$ & $1,00(0,82)$ & $0,00(0,00)$ & $0,00(0,00)$ \\
MCTP 01 & $3,33(1,51)$ & $3,50(1,97)$ & $1,83(0,75)$ & $1,33(1,03)$ & $0,50(0,55)$ & $1,00(0,63)$ & $0,50(0,55)$ & $0,50(0,84)$ \\
MCTP 02 & $4,83(2,32)$ & $4,33(3,50)$ & $1,67(0,52)$ & $1,33(1,03)$ & $1,50(0,84)$ & $1,67(1,21)$ & $0,50(0,84)$ & $0,33(0,52)$ \\
\hline
\end{tabular}

Media (DS) Variables dependientes por Grupo de análisis 
la variable "TPPCEs" (Figura 2) mostró una tendencia a la disminución del tiempo en todos sus grupos, expresando diferencias significativas en el grupo 1 en sus dos órdenes ( $M C$ 01, $P=0,031 ; M C$ 02, $P=0.009$ ), en el grupo 2 en sus dos órdenes (MCP 01, $P=0.005 ;$ MCP 02, $P=0.005$ ), en el grupo 3 en su orden 1 (MCB 01, $P=0.002$ ), en el grupo 4 en sus dos órdenes (MCBP 01, $P=0.008$; MCBP 02, $P=0.003$ ), en el grupo 5 en su orden 1 (MCT 01, $P=0.002$ ), y en el grupo 6 en su orden 1 (MCTP 01, $P=0.011$ ); todos con un $\mathrm{P}$ valor menor al encontrado en el grupo control.

Con respecto a la variable "TTFPm", se presentó una tendencia a la disminución

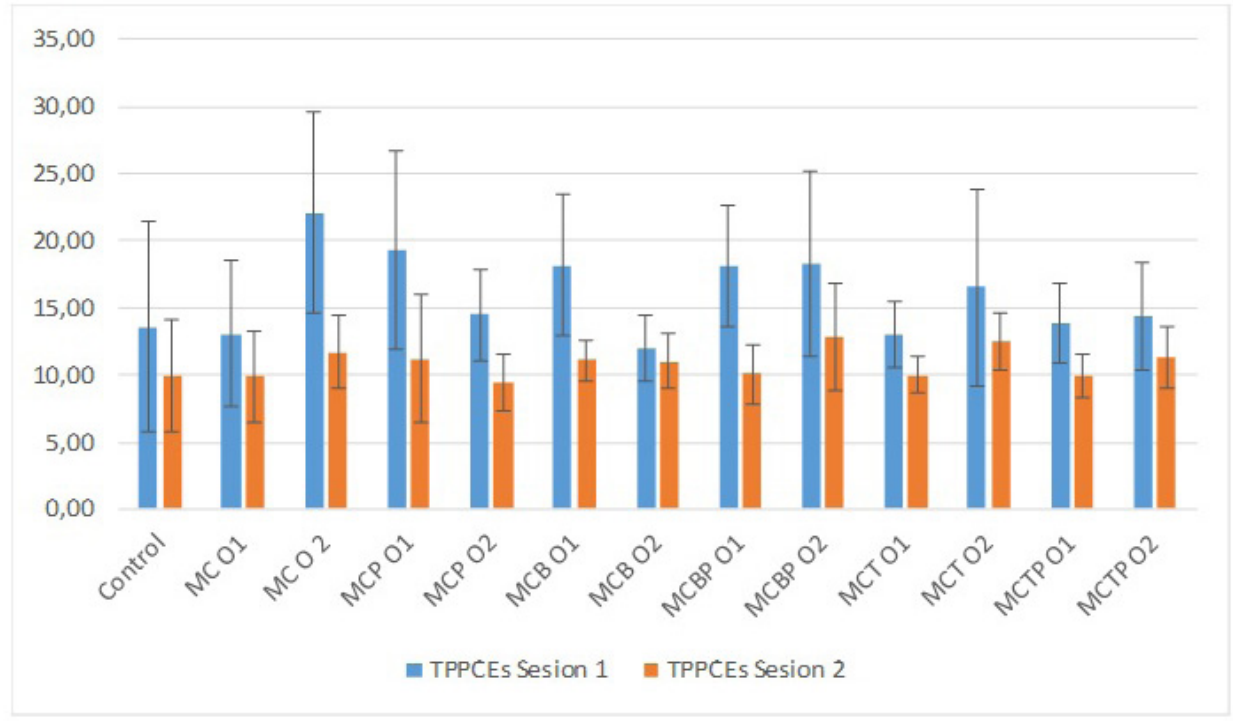

Figura 2. Medias y desviaciones estándar de "TPPCEs"

en el tiempo que tardaban los participantes en realizar la tarea experimental en todos los grupos durante la segunda sesión, independiente del orden de presentación (Figura 3). Sin embargo, no en todos los órdenes se presentaron diferencias significativas entre sesiones. El grupo 1 mostró diferencias significativas en su segundo orden ( $M C$ 02, $P=0.002$ ), mientras el grupo 2 mostró diferencias en sus dos órdenes (MCP 01, P=0.008; MCP 02, P=0.047); el grupo 3 (MCB) y el grupo 5 (MCT) mostraron diferencias únicamente en su primer orden (MCB 01, $P=0.003$; MCT 01, $P=0.018$ ), mientras los grupos 4 (MCBP) y 6 (MCTP) mostraron diferencias en sus dos órdenes de presentación (MCBP 01, $P=0.005$; MCBP 02, P=0.005; MCTP 01, $P=0.024 ;$ MCTP 02, $P=0.004$ ).

Se destaca que solamente los grupos con la variable placebo presentaron diferencias en sus dos órdenes de presentación, los grupos con la variable binaural sin placebo presentaron diferencias únicamente en el orden 1 y el grupo con música clásica sin placebo ni binaural presentó diferencias únicamente en su orden 2. Sin embargo, en el análisis intersujeto no se encontraron significancias con respecto a la ausencia o presencia de información placebo en la comparación entre sesiones.

Se encontró, además, significancia en la comparación de un factor entre los dos órdenes del grupo 1 en su primera sesión ( $P=0.003)$, mostrando un menor rango de tiempo con el estímulo de música clásica que sin él, lo cual puede explicarse por el desempeño del grupo 1 en su orden 2; al ser los resultados de la primera sesión los que se están comparando, todos los resultados de orden 2 corresponden a una primera aplicación de la tarea sin presencia de la Vi, por lo que la comparación de un 
grupo en su orden 1 con cualquier grupo en su orden 2 sería válida para corroborar el $p$ valor y rechazar la hipótesis nula de igualdad de medias. Al comparar el grupo 1 en su orden 1 con otros grupos en su orden de presentación 2 no se encuentran diferencias significativas, por lo que no se puede aceptar la hipótesis alternativa de que la diferencia entre las medias se da por la presencia de la Vi.

En cuanto a la variable "Porcentaje de Ecuaciones Acertadas" (Figura 4) se encontró

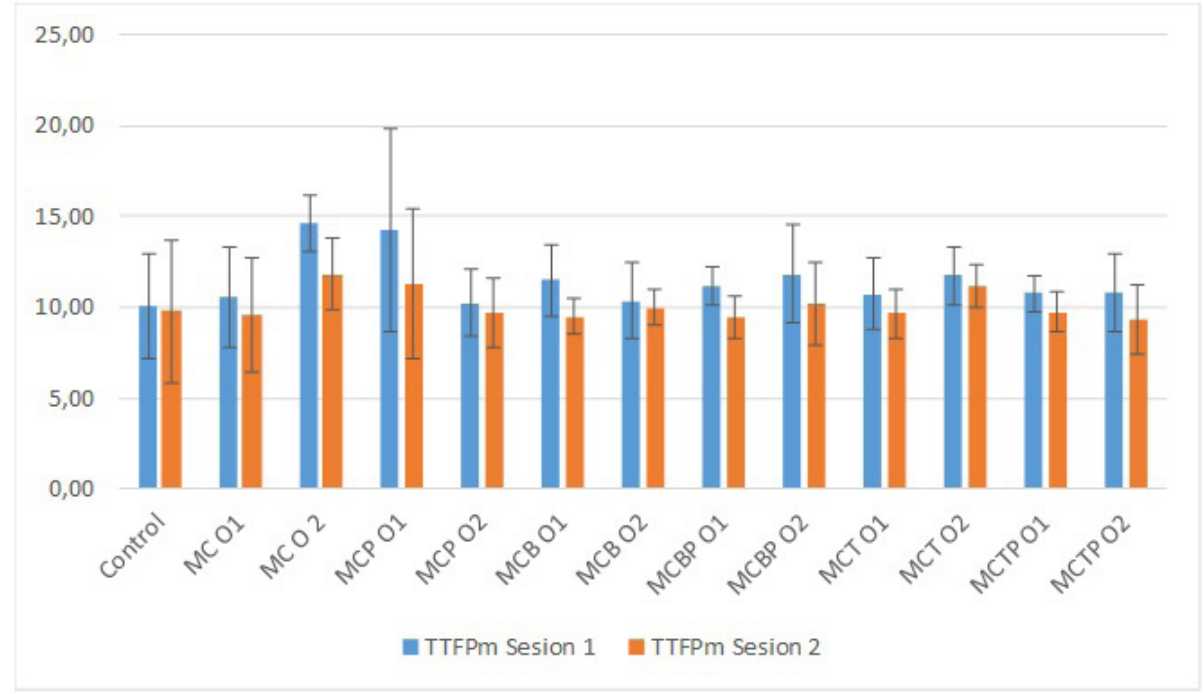

Figura 3. Medias y desviaciones estándar de "TTFPm"

una diferencia significativa en el grupo 2 en su orden 2 (MCP 02, P=0.034), la cual muestra un deterioro en el porcentaje de ecuaciones acertadas entre la primera y la segunda sesión. En el análisis con factor intersujeto se encontraron diferencias significativas entre los grupos 1 y 2 en su orden 2 con respecto a la ausencia/presencia de información placebo (MC 02 - MCP 02, P=0.049), viéndose un mayor deterioro entre sesiones durante la aplicación del estímulo de música clásica acompañada con placebo; sin embargo, se sigue presentando un mejor puntaje en MCP 02 tanto en la primera como en la segunda sesión. Debido a que el deterioro sólo se observó en este grupo y, por lo contrario, en los demás grupos del mismo orden que también fueron acompañados por información placebo se observó un aumento en el porcentaje de aciertos, aunque no significativo, no puede establecerse una relación directa entre el factor placebo y el deterioro en el puntaje de ecuaciones.

Adicionalmente, se presentaron significancias en las comparaciones de un factor entre los dos órdenes del grupo 1 tanto en su primera sesión $(P=0.011)$ como en la segunda $(P=0.005)$, y entre los dos órdenes de la segunda sesión del grupo 6 $(P=0.017)$, mostrándose mejores puntajes en ambas sesiones en el orden 1 del grupo 1 y un mejor puntaje en la segunda sesión en el orden 2 del grupo 6. En el caso del grupo 1, esto se explicaría por el mismo rendimiento de los grupos, los cuales no presentaron diferencias destacables entre la primera y la segunda sesión, rechazando con esto una posible influencia de estimulación sonora presentada. En el caso del grupo 6 no puede atribuirse dicha mejoría a la presentación del estímulo debido a que, durante la primera sesión, el orden 1, que en este instante fue el grupo que recibió la estimulación sonora, marcó un menor puntaje que el grupo en su orden 2.

Con respecto a las variables de recuerdo, en la variable "Sets completos" (Figura 5), 


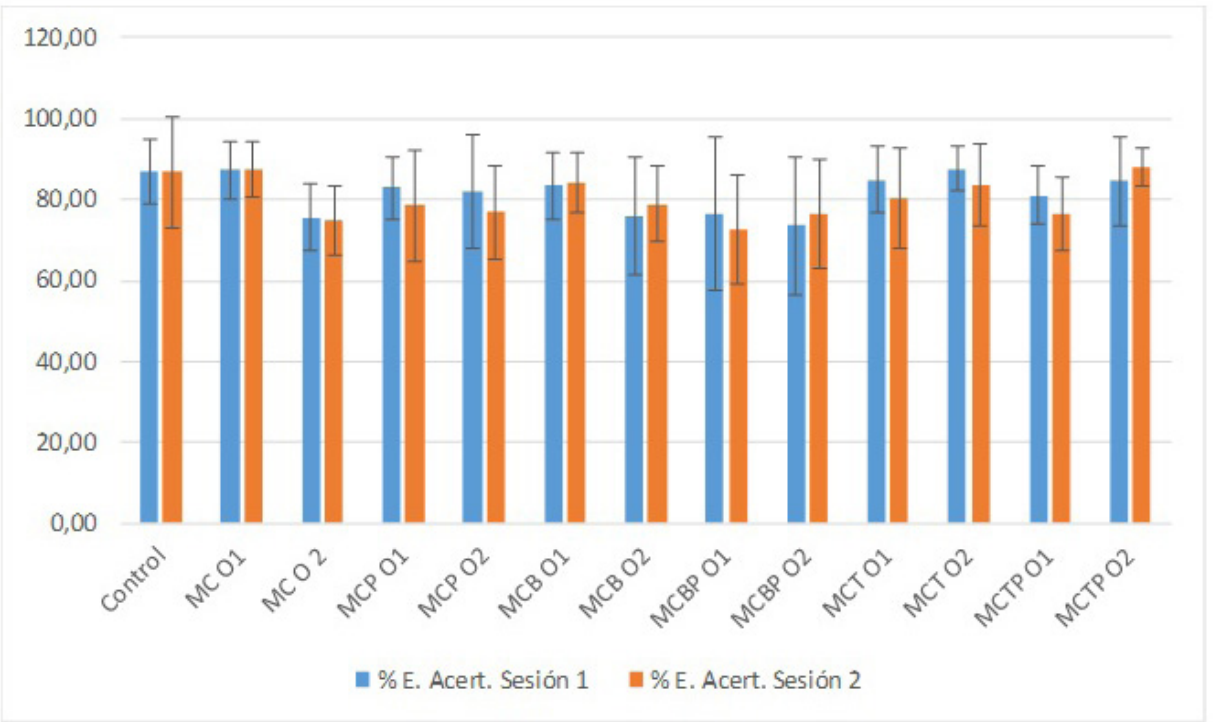

Figura 4. Medias y desviaciones estándar de "Porcentaje de Ecuaciones Acertadas"

se observaron diferencias significativas en el análisis intrasujeto del grupo 3 en su orden 1 (MCB 01, P=0.013), mostrando una mejoría en la puntuación entre la primera y la segunda sesión de la tarea experimental; la cual se relaciona con significaciones encontradas en los subniveles "Sets 3" $(P=0.014)$ y "Sets 4" $(P=0.014)$ del mismo grupo, presentando en ambos una mejoría entre sesiones (Figura 6). Sin embargo, estos cambios no afectan la variable "OSPAN" lo suficiente para alcanzar diferencias significativas en el p valor, que en este caso es igual 0.05; el cual no se tomará como representativo en la medida de que no se observaron significancias en la variable "Letras recordadas" que se puedan relacionar con este puntaje. Esta variable manifestaría concretamente afectaciones en el recuerdo libre de toda la prueba por lo que, al no encontrar significancias en esta, no puede hacerse referencia a una mejoría en el recuerdo global de la tarea. Lo que sí puede señalarse es una mejoría en el recuerdo de conjuntos de datos que en este caso no superan los 4 elementos y que se relacionan con una exposición a la variable independiente durante una primera sesión.

Las desviaciones de la variable superan su media en el grupo 5 de orden 1 (MCT 01) y

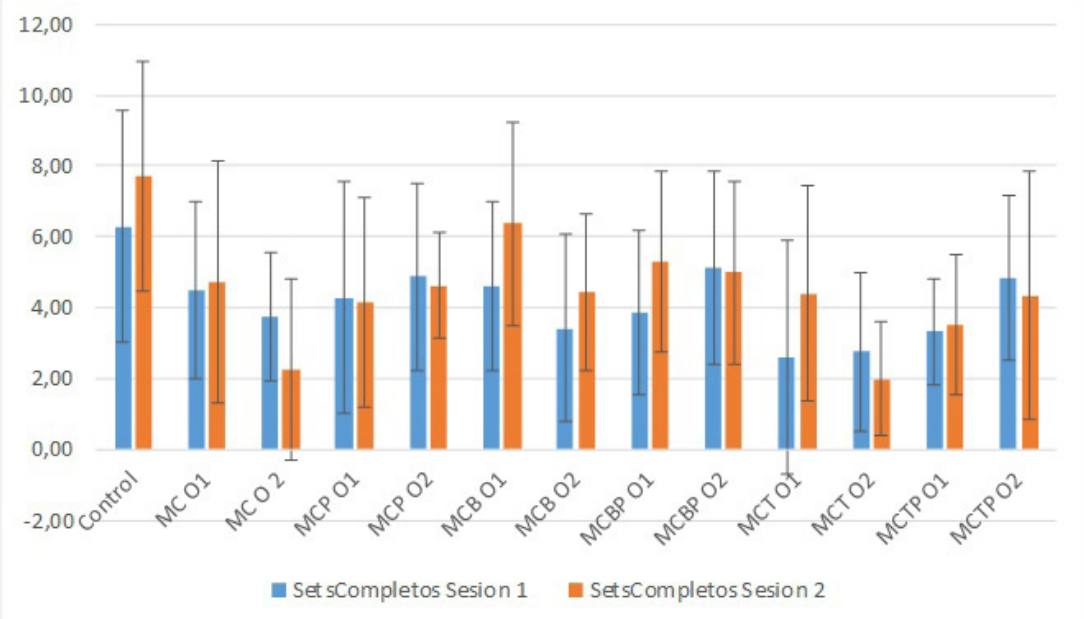

Figura 5. Medias y desviaciones estándar de "Sets Completos" 
el grupo 1 de orden 2 (MC 02), presentándose un sesgo en la distribución de los datos de dichos grupos, el cual se explica por el sistema de calificación de sets (ver 2.1.2) volviendo a la variable más susceptible de Asimetricidad en todos los sub puntajes correspondientes a "Sets Completos".

No se encontraron diferencias en el análisis con factor intersujeto, con respecto a
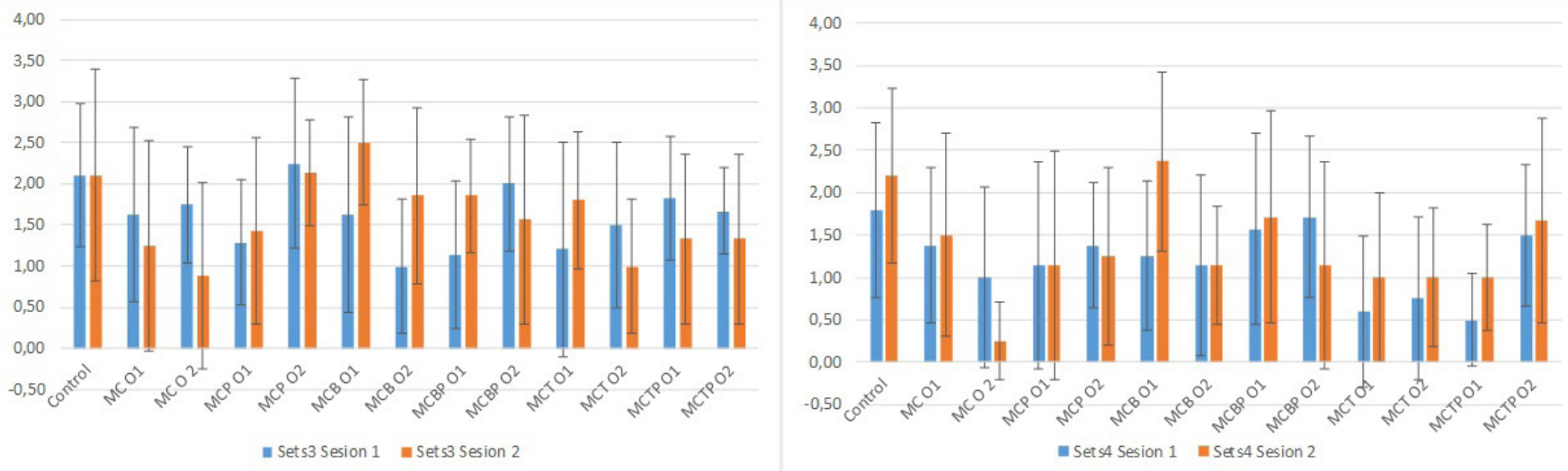

Figura 6. Medias y desviaciones estándar de "Sets 3" y "Sets 4", relacionados con la variable "Sets Completos" en el P valor presentado en MCB 01.

la ausencia o presencia de información placebo, ni en la comparación entre órdenes durante una misma sesión en ninguna de las variables de recuerdo. Razón por la cual solo puede hablarse de una ventaja del orden 1, presentación de la Vi en la primera sesión, sobre el orden 2, presentación de la Vi en la segunda sesión, en el grupo 3, estimulado con binaural de onda beta sin placebo (MCB), siendo este el único grupo que presentó significancias en las variables de recuerdo.

\section{Discusión}

Los resultados obtenidos en este estudio señalan que no hay un efecto de los sonidos binaurales de onda beta o theta sobre el desempeño en la evocación total de elementos de la tarea de memoria de trabajo computarizada seleccionada, la cual consistió en el recuerdo de sets de letras que se fueron presentando de forma intercalada con la presentación de pruebas matemáticas que funcionaban como prueba de interferencia; esto debido a que no se encontraron diferencias significativas en las variables de recuerdo global, OSPAN total y letras recordadas. Este hallazgo, que va en contraposición con resultados de estudios anteriores, puede explicarse por la tarea de recuerdo aplicada, la cual exigía la retención y evocación de letras en orden directo sin ayuda alguna, en relación con la tarea de interferencia; al estar menos relacionadas la tarea de recuerdo con la tarea de interferencia se vuelve más difícil la tarea de evocación directa que pedía la prueba, causando, por ende, un mayor esfuerzo cognitivo en la evocación.

Diversos estudios que han encontrado diferencias significativas intergrupales en sus respectivas tareas de recuerdo utilizaron tareas de reconocimiento en las que se solicitó a los participantes, divididos a razón de una determinada estimulación sonora, identificar los elementos presentados dentro de una lista de elementos más grande (Beauchene et al. 2016; Beauchene, Abaid, Moran, Diana, \& Leonessa, 2017; Garcia-Argibay, Santed, \& Reales, 2017). Esta elección en la tarea se observa también 
Pág 46

Los resultados obtenidos en este estudio señalan que no hay un efecto de los sonidos binaurales de onda beta 0 theta sobre el desempeño en la evocación total de elementos de la tarea de memoria de trabajo computarizada seleccionada, la cual consistió en el recuerdo de sets de letras que se fueron presentando de forma intercalada con la presentación de pruebas matemáticas que funcionaban como prueba de interferencia; esto debido a que no se encontraron diferencias significativas en las variables de recuerdo global, OSPAN total y letras recordadas. recuerdo implica seleccionar las letras de cada set en el orden en que se presentan, de una pantalla de recuperación que muestra las letras presentadas durante el set y más letras de interferencia. En estas tareas prima la memoria por reconocimiento, pues el sujeto se encarga de reconocer los elementos que le habían sido presentados en lugar de evocarlos de manera inmediata (Bakker-Marshall et al., 2018). Contrario a lo anterior, la prueba utilizada en el presente estudio exige una evocación directa de los valores presentados, provocando que el individuo recurra al almacenamiento a corto plazo de los sets de letras, es decir, sin la asociación icónica entre la memoria inmediata y las opciones que evoquen el recuerdo.

Si se observan los requerimientos cognitivos de estas tareas de recuerdo y se traslapan a un nivel neuroanatómico, se puede encontrar en la región parietal una explicación funcional de las razones por las cuales cuando la tarea de interferencia es una tarea matemática, la evocación por comparación o reconocimiento de elementos resulta mucho más asequible que el recuerdo inmediato de los mismos; y es que tanto la asociación por comparación de elementos, que requiere de la elaboración mental de la imagen relacionada con lo que se está viendo (Qin et al., 2004), como el desempeño en las tareas de aproximación aritmética (Dehaene, 1997) están fuertemente relacionadas con la activación de dicha región (Radford \& André, 2009).

Si bien es cierto que el desempeño óptimo de la memoria de trabajo requiere de la interconexión funcional del córtex prefrontal con regiones parietales, temporales o de los colículos superiores (Kamigaki \& Dan, 2017), la tarea de recuerdo o evocación se facilita mucho más si está relacionada con la tarea de interferencia. De esta manera, al evaluar la evocación por reconocimiento y comparación de elementos con una interferencia por aproximación aritmética, se estaría dando una relación entre el córtex prefrontal como sistema ejecutivo y el lóbulo parietal como área de asociación y procesamiento por imaginería, entendiendo esta última como la elaboración mental de una imagen para la realización de una tarea (Anderson, Qin, Sohn, Stenger, \& Carter, 2003; Honey, et al., 2002; Qin, et al., 2004), necesarios en este caso tanto en la tarea de interferencia como en la tarea de recuerdo. Esto implicaría un mayor ahorro de recursos en cuanto a que el sistema de procesamiento de la tarea se engloba en una misma relación cortical, contrario a una tarea de recuerdo directo en la que, además de la activación en la conexión prefrontal-parietal, se requiere también de la activación de zonas occipito-temporales que evoquen el recuerdo sin ayuda de un medio externo asociado (Brysbaert, Cai, \& Van Der Haegen, 2012, citado en Ivanova, 2017; Solís \& López-Hernández, 2009).

Ahora, debido al hecho de que se hayan presentado diferencias significativas en el grupo control y los demás grupos ya especificados en el TPPCEs, grupos 1, 2 y 4 en ambos órdenes, y grupos 3, 5, y 6 en su primer orden, y que este tiempo promedio se relaciona con el Tiempo total de la fase de prueba, no podría establecerse concretamente que los resultados obtenidos en el TTFPm se deban a la presentación de los estímulos sonoros ya tengan carácter binaural o no; sin embargo, debido a que solo en los grupos que fueron acompañados por la variable placebo se presentaron diferencias significativas en el orden de presentación 2, se puede sugerir que la variable placebo utilizada tuvo una influencia sobre la velocidad de desarrollo de la tarea matemática, facilitando la velocidad de evocación de la tarea, o al menos la percepción de un mejor desenvolvimiento en el desarrollo de ésta, en cuanto a un procesamiento posterior. Esto se traduciría en un reforzamiento de la sensación de aprendizaje por mecanización que se explica con las conclusiones de Qin et al. (2004), según las cuales el proceso de resolución matemática, luego de la práctica y el aprendizaje, dependería 
Debido a que solo en los grupos que fueron acompañados por la variable placebo se presentaron diferencias significativas en el orden de presentación 2 , se puede sugerir que la variable placebo utilizada tuvo una influencia sobre la velocidad de desarrollo de la tarea matemática, facilitando la velocidad de evocación de la tarea, o al menos la percepción de un mejor desenvolvimiento en el desarrollo de ésta, en cuanto a un procesamiento posterior. más de la mecanización de operaciones sucesivas que del procesamiento por imaginería.

En cuanto a los cambios observados en la variable "Sets Completos", significancias en el primer orden del grupo 3, que se deben a las significancias en los subniveles "Sets de 3" y "Sets de 4", a simple vista podrían corroborar las aseveraciones de Scholz, Schneider y Rose (2017) cuando refieren que el rango de frecuencia beta puede asociarse con un mejor rendimiento de la memoria, que en este caso se vería reflejado en la recuperación de conjuntos de hasta cuatro elementos; sin embargo, al no presentarse diferencias significativas en el grupo 4 del mismo orden (MCBP 01), el cual tiene la misma estimulación binaural de frecuencia beta, este resultado se torna ambiguo.

Finalmente, la ausencia de significancias en los grupos 5 y 6 , estimulados con el sonido binaural theta, puede explicarse al considerar que las relaciones encontradas entre la activación de la banda theta con procesos de memoria se dan con procesos de consolidación de información o con tareas de recuerdo de elementos ya consolidados (Bakker-Marshall et al., 2018; Düzel, Penny, \& Burgess, 2010; Scholz, Schneider, \& Rose, 2017), requisito que no se cumple en esta tarea al evaluar elementos de recuerdo en la memoria a corto plazo. La banda beta, por otro lado, tiende a relacionarse con el mantenimiento del conjunto sensorio-motor actual y la comunicación entre áreas prefrontales y postero-parietales (Engel \& Fries, 2010; Khanna \& Carmena, 2005), que tienen mayor relación con el funcionamiento ejecutivo (Kamigaki \& Dan, 2017). No obstante, como ya se señaló, los resultados encontrados son ambiguos al no presentarse en los dos grupos del mismo orden estimulados con el sonido binaural beta, por lo que no se puede establecer relación directa entre la Vi y los resultados, lo cual requeriría estudios posteriores.

\section{Conclusiones}

Según los hallazgos de la presente investigación, no se presenta una relación directa entre la estimulación por música clásica transformada a sonido binaural de onda beta y theta, con mejoras en el procesamiento y especialización de la información en tareas de interferencia matemática, en las que se ve implicada la memoria de trabajo.

La presencia de información placebo, dirigida al aumento de expectativas con respecto al rendimiento de tareas implicadas en procesos de memoria, puede relacionarse con una disminución en el tiempo de presentación de la tarea de memoria de trabajo por interferencia matemática sin implicar una mayor eficacia en ésta. Este efecto aún debe estudiarse más, cobrando especial importancia en el desarrollo de las formas de intervención por estimulación cognitiva en los ámbitos clínico o educativo, y en la percepción de mejoría o bienestar de los individuos.

A pesar de que estudios anteriores a éste mostraron una estimulación efectiva con respecto al rendimiento en las tareas de memoria de trabajo utilizadas, este análisis permite observar una brecha teórica y metodológica dentro de los mismos que obliga a pensar en la especificidad del proceso que se evalúa en una tarea experimental; si bien no se niega la implicación de la memoria de trabajo dentro de las tareas de interferencia utilizadas en todos los estudios mencionados y en este mismo, es necesario especificar tanto el tipo de interferencia como la forma de recuperación de la información al aunarlos al efecto de algún tipo de estimulación. Aunque sea cierto que el mecanismo tripartito de la memoria de trabajo puede seguir siendo el mismo, los procesos sobre los cuales trabaja pueden ser distintos de acuerdo con la 
Pág 48

La presencia de información placebo, dirigida al aumento de expectativas con respecto al rendimiento de tareas implicadas en procesos de memoria, puede relacionarse con una disminución en el tiempo de presentación de la tarea de memoria de trabajo por interferencia matemática sin implicar una mayor eficacia en ésta. tarea exigida y el estímulo presentado, lo cual implica que el efecto de una variable independiente puede así mismo variar de acuerdo con estos o más factores.

La necesidad de un estudio sistemático que permita visualizar la especificidad del efecto de las ondas binaurales en procesos mnésicos dentro de tareas experimentales y la metodología a utilizar respecto a su presentación, se ha hecho tangible en estudios anteriores; el presente estudio refuerza dicha necesidad y marca una línea de avance con respecto a este tema. Se recomienda realizar estudios teniendo en cuenta el momento de la estimulación y la importancia del requerimiento cognitivo que exige la tarea que utilicen.

Finalmente, en función de contrastar los resultados y las valoraciones obtenidas en este estudio, en especial las consideraciones sobre el recuerdo inmediato y su diferenciación con la evocación por asociación y comparación de elementos, se recomienda desarrollar de nuevo esta metodología con una tarea de interferencia cuya porción de recuerdo implique la evocación del elemento por asociación y comparación, como el AOSPAN original; como resultado se esperaría un mejor desempeño en la tarea de memoria. Esto reforzaría cualquier consideración previa a la aplicación de este tipo de estimulación sobre población clínica, que debería ser una población objeto en este tipo de estudios, pero sobre los cuales se debe trabajar minimizando los factores de error en el efecto de los factores independientes a estudiar.

\section{Agradecimientos}

Al Dr. Pedro Gonzales Velasco, por su ayuda en la transformación del estímulo sonoro seleccionado a los binaurales utilizados en la fase experimental.

A Ana María Daza, por su ayuda en la selección del diseño de análisis de los datos obtenidos y su constante apoyo en la redacción y revisión general del artículo.

\section{Referencias}

Aguilar, M. (2004). Memoria. En Mestre, J. M., y Palmero F. (Eds.). Procesos Psicológicos Básicos: Una guía académica para los estudios en psicopedagogía, psicología y pedagogía (pp. 103-136). Madrid: McGraw-Hill.

Anderson, J. R., Qin, Y., Sohn, M. H., Stenger, V. A., \& Carter, C. S. (2003). An information-processing model of the BOLD response in symbol manipulation tasks. Psychonomic Bulletin and Review, 10(2), 241-261. doi: https://doi.org/10.3758/BF03196490

Atkinson, R., \& Shiffrin, R. (1971). The control of short-term memory. Scientific American, 225(2), 82-90.

Baddeley, A., \& Hitch, G. (1974). Working memory. The psychology of learning and motivation, 8, 47-89.

Bakker-Marshall, I., Takashima, A., Schoffelen, J.-M., van Hell, J. G., Janzen, G., \& McQueen, J. M. (2018). Theta-band Oscillations in the Middle Temporal Gyrus Reflect Novel Word Consolidation. Journal of Cognitive Neuroscience, 30(5), 621-633. doi: https://doi. org/10.1162/jocn a 01240

Balderas, I. Ramírez, V., \& Bermúdez, F. (2004). Cambios morfológicos asociados a la memoria. Revista de Neurología, 38(10), 944-948. Recuperado de: https://www. researchgate.net/profile/Federico Bermudez-Rattoni/publication/8530785 Memory-linked morphological changes/links/551d995f0cf2a2d9e13afe4a.pdf

Beauchene, C., Abaid, N., Moran, R., Diana, R., \& Leonessa, A. (2016). The Efect of Binaural Beats on Visuospatial Working Memory and Cortical Connectivity. Plos One, 11(11), 1-20. doi: https://doi.org/10.1371\%2Fjournal.pone.0166630

Beauchene, C., Abaid, N., Moran, R., Diana, R. A., \& Leonessa, A. (2017). The effect of 
binaural beats on verbal working memory and cortical connectivity. Journal of Neural Engineering, 14(2). doi: https://doi.org/10.1088/1741-2552/aa5d67

Becher, A. Höhne, M. Axmacher, N. Chaieb, L. Elger, C., \& Fell, J. (2014). Intracranial electroencephalography power and phase synchronization changes during monaural and binaural beat stimulation. European Journal of Neuroscience, 41(2), 254-263. doi: https://doi.org/10.1111/ejn.12760

Benedetti, F., Mayberg, H. S., Wager, T. D., Stohler, C. S., \& Zubieta, J.-K. (2005). Neurobiological Mechanisms of the Placebo Effect. Journal of Neuroscience, 25(45), 10390-10402. doi: https://doi.org/10.1523/JNEUROSCI.3458-05.2005

Boot, W. R., Simons, D. J., Stothart, C., \& Stutts, C. (2013). The Pervasive Problem with Placebos in Psychology: Why Active Control Groups Are Not Sufficient to Rule Out Placebo Effects. Perspectives on Psychological Science, 8(4), 445-454. doi: https://doi.org/10.1177/1745691613491271

Carasatorre, M. Ramírez, V., \& Díaz, S. (2016). Plasticidad sináptica estructural en el hipocampo inducida por la experiencia espacial y sus implicaciones en el procesamiento de información. Neurología, 31(8), 543-549. doi: https://doi. org/10.1016/j.nrl.2012.12.005

Carruthers, P (2013). La Evolución de la Memoria de Trabajo. Ludus Vitalis, 21(40), 99124. Recuperado de: http://www.ludus-vitalis.org/ojs/index.php/ludus/article/ view/41/41

Castillo, G. García, E., \& Castillo, S. (2014). Sincronización cerebral con estimulación transcraneal. Acta Neurológica Colombiana, 30(2), 103-107. Recuperado de: http:// www.acnweb.org/images/stories/pdf/acta2014/acta-2014 30 103-107.pdf

Chaieb, L., Höhne, M., Staresina, B., Elger, C. E., Hampel, K., Surges, R., \& Fell, J. (2017). Modulation of long-term memory using auditory beat stimulation. Brain Stimulation, 10(2), 360. doi: https://doi.org/10.1016/j.brs.2017.01.061

Clemenson, G. D., Gage, F. H., \& Stark, C. E. L. (2018). Environmental Enrichment and Neuronal Plasticity. In M. V. Chao (Ed.), The Oxford Handbook of Developmental Neural Plasticity (Vol. 85, pp. 283-284). Oxford University Press. doi: https://doi. org/10.1093/oxfordhb/9780190635374.013.13

Córdoba, A., Albert, J., \& López, S. (2010). Potenciación a largo plazo en la corteza humana. Revista de Neurología, 51(6), 367-374. Recuperado de doi: http://www. neurologia.com/articulo/2009616

D'Esposito, M., \& Postle, B. R. (2015). The Cognitive Neuroscience of Working Memory. Annual Review of Psychology, 66(1), 115-142. doi: https://doi.org/10.1146/annurev-psych-010814-015031

de la Fuente-Fernandez, R., Ruth, T. J., Sossi, V., Schulzer, M., Calne, D. B., \& Stoessl, A. J. (2001). Expectation and Dopamine Release: Mechanism of the Placebo Effect in Parkinson's Disease. Science, 293(5532), 1164-1166.

Dodla, R., \& Wilson, C. J. (2017). Effect of Phase Response Curve Shape and Synaptic Driving Force on Synchronization of Coupled Neuronal Oscillators. Neural Computation, 29(7), 1769-1814. doi: https://doi.org/10.1162/NECO a 00978

Düzel, E., Penny, W. D., \& Burgess, N. (2010). Brain oscillations and memory. Current Opinion in Neurobiology, 20(2), 245-257. doi: https://doi.org/10.1016/j.conb.2010.01.004

Engel, A. K., \& Fries, P. (2010). Beta-band oscillations-signalling the status quo? Current Opinion in Neurobiology, 20(2), 156-165. doi: https://doi.org/10.1016/j. conb.2010.02.015

Etchepareborda, M., \& Abad-Mas, L. (2005). Memoria de trabajo en los procesos básicos de aprendizaje. Revista de Neurología, 40(supl 1), S79-S83. Recuperado de: http:// www.neurologia.com/articulo/2005078

Fajardo, A., \& Guzman, A. (2016). Neurofeedback, aplicaciones y eficacia. Interdiscipli- 
naria, 33(1), 81-93. Recuperado de: http://www.scielo.org.ar/pdf/interd/v33n1/ v33n1a05.pdf

Figueiras, R., Magariños, C., Regidor, l., del Álamo,R., Cabañes, L., \& Gómez, M. (2009). Estimulación cerebral profunda: 12 años de experiencia y 250 pacientes intervenidos con un seguimiento de más de un año. Revista de Neurología, 49(10), 511-516. Recuperado de: http://www.neurologia.com/articulo/2009055

Freigni, F., \& Pascual-Leone, A. (2007). Technology Insight: noninvasive brain stimulation in neurology-perspectives on the therapeutic potential of rTMS and tDCS. Nature Reviews Neurology, 3(7), 383-393. doi: https://doi.org/10.1038/ncpneuro0530

Gao, X., Cao, H., Ming, D., Qi, H., Wang, X., Wang, X., \& Peng, Z. (2014), Analysis of EEG Activity in Response to Binaural Beats with Different Frequencies. International Journal of Psychophysiology, 94(3), 399-406. doi: https://doi.org/10.1016/i.ijpsycho.2014.10.010

Garcia-Argibay, M., Santed, M. A., \& Reales, J. M. (2017). Binaural auditory beats affect long-term memory. Psychological Research, 83(6) 1124-1136. doi: https://doi. org/10.1007/s00426-017-0959-2

Gonzales, P. (2013). Influencia de la estimulación sonora binaural en la generación de ondas cerebrales: Estudio electroencefalográfico. (Tesis Doctoral) Universidad Complutense de Madrid. Madrid, España. Recuperado de: http://eprints.ucm. es/21680/1/T34524.pdf

Goto, Y., Yang, C., \& Otani, S. (2011). Plasticidad sináptica funcional y disfuncional en la corteza prefrontal: papel en los trastornos psiquiátricos. Psiquiatría Biológica, 18(1), 18-27. doi: https://doi.org/10.1016/j.psiq.2009.08.001

Gutiérrez, F., García, J., Carriedo, N., Vila, J., \& Luzón, J. (2005). Dos pruebas de Amplitud de Memoria Operativa para el Razonamiento. Cognitiva, 17(2), 183-210. Recuperado de: http://portal.uned.es/pls/portal/docs/PAGE/UNED MAIN/LAUNIVERSIDAD/UBICACIONES/02/DOCENTE/M. NURIA CARRIEDO LOPEZ/PUBLICACIONES/DOS\%20PRUEBAS\%20DE\%20AMPLITUD\%20DE\%20MEMORIA\%20 OPERATIVA.PDF

Honey, G. D., Fu, C. H. Y., Kim, J., Brammer, M. J., Croudace, T. J., Suckling, J., ... Bullmore, E. T. (2002). Effects of verbal working memory load on corticocortical connectivity modeled by path analysis of functional magnetic resonance imaging data. NeuroImage, 17(2), 573-582. doi: https://doi.org/10.1016/S1053-8119(02)91193-6

Ivanova, O. (2017). Fundamentos neurocognitivos del procesamiento lingüístico. Estudios de Lingüística Aplicada, 35(66), 235-261. doi: https://doi.org/10.22201/ enallt.01852647p.2017.66.838

Kamigaki, T., \& Dan, Y. (2017). Delay activity of specific prefrontal interneuron subtypes modulates memory-guided behavior. Nature Neuroscience, 20(6), 854-863. doi: https://doi.org/10.1038/nn.4554

Kennerly, R. C. (1994). An empirical investigation into the effect of beta frequency binaural beat audio signals on four measures of human memory (tesis de Maestría) West Georgia College. Georgia, EEUU. Recuperado de: http://www.nueva-mente. com/Research/13.htm

Khanna, P., \& Carmena, J. M. (2015). Neural oscillations: Beta band activity across motor networks. Current Opinion in Neurobiology, 32, 60-67. doi: https://doi. org/10.1016/j.conb.2014.11.010

Leff, P., Retana, I. Arias, A., Zavala, E., Loria, F. Pavón, L., \& Antón, B. (2004). Understanding the Neurobiological Mechanisms of Learning and Memory: Cellular, Molecular and Gene Regulation Implicated in Synaptic Plasticity and Long-Term Potentiation. Part IV A. Salud Mental, 27(2), 39-54. Recuperado de: http://new.medigraphic. $\mathrm{com} / \mathrm{cgi}$-bin/resumenl.cgi?IDARTICULO $=16543$

Llacanfil, N. (2013). Efectos de los infrasonidos en la conducta humana. (Tesis de 
pregrado) Universidad Austral de Chile. Valdivia, Chile. Recuperado de: http:// cybertesis.uach.cl/tesis/uach/2013/bmfcil791e/doc/bmfcil791e.pdf

Morgado, I. (2005). Psicobiología del aprendizaje y la memoria: fundamentos y avances recientes. Revista de Neurología, 40(5), 289-297. Recuperado de: http://www.neurologia.com/articulo/2005004

Nicoll, R. A. (2017). A Brief History of Long-Term Potentiation. Neuron, 93(2), 281-290. doi: https://doi.org/10.1016/j.neuron.2016.12.015

Ortega Loubon, C., \& Franco, J. (2010). Neurofisiología del aprendizaje y la memoria. Plasticidad Neuronal. Archivos de medicina, 6(1), 1-7. Recuperado de http://www. archivosdemedicina.com/medicina-de-familia/neurofisiologa-del-aprendizaje-y-la-memoria-plasticidad-neuronal.pdf

Peirce, J. W. (2007). PsychoPy - Psychophysics software in Python. Journal of Neuroscience Methods, 162(1-2), 8-13. doi: https://doi.org/10.1016/j.jneumeth.2006.11.017

Pluta, R. (2011). Deep Brain Stimulation. JAMA, 305(7), 732-732. Recuperado de: http://jamanetwork.com/journals/jama/fullarticle/645662? resultClick=1

Qin, Y., Carter, C. S., Silk, E. M., Stenger, V. A., Fissell, K., Goode, A., \& Anderson, J. R. (2004). The change of the brain activation patterns as children learn algebra equation solving. Pnas, 101(15), 5686-5691. doi: https://doi.org/10.1073/ pnas.0401227101

Radford, L., \& André, M. (2009). Cerebro, Cognición y Matemáticas Brain. Revista Latinoamericana de Investigación En Matemática Educativa, 12, 215-250.

Scholz, S., Schneider, S. L., \& Rose, M. (2017). Differential effects of ongoing EEG beta and theta power on memory formation. PLOS ONE, 12(2), 1-18. doi: https://doi. org/10.1371/journal.pone.0171913

Seifi Ala, T., Ahmadi-Pajouh, M. A., \& Nasrabadi, A. M. (2018). Cumulative effects of theta binaural beats on brain power and functional connectivity. Biomedical Signal Processing and Control, 42, 242-252. doi: https://doi.org/10.1016/j. bspc.2018.01.022

Solís, H., \& López-Hernández, E. (2009). Neuroanatomía funcional de la memoria. Archivos de Neurociencias (México), 14(3), 176-187.

Stelzer, F. Andés, M. Canet-Juric, L., \& Introzzi, I. (2016). Memoria de Trabajo e Inteligencia Fluida. Una Revisión de sus Relaciones. Acta de Investigación Psicológica, 6(1), 2302-2316. doi: https://doi.org/10.1016/S2007-4719(16)30051-5

Turner, M., \& Engle, R. (1989). Is Working Memory Capacity Task Dependent?. Journal of Memory and Language. 28, 127-154. doi: https://doi.org/10.1016/0749$\underline{596 \times(89) 90040-5}$

Unsworth, N. Heitz, R. Schrock, J., \& Engle, R. (2005). An automated version of the operation span task. Behavior Research Methods, 37(3), 498-505. Recuperado de: https://link.springer.com/article/10.3758/BF03192720

Vernon, D., Peryer, G., Louch, J., \& Shaw, M. (2014). Tracking EEG changes in response to alpha and beta binaural beats. International Journal of Psychophysiology, 93(1), 134-139. doi: https://doi.org/10.1016/j.ijpsycho.2012.10.008

Wahbeh, H., Calabrese, C., Zwickey, H., \& Zajdel, D. (2007). Binaural Beat Technology in Humans: A Pilot Study to Assess Neuropsychologic, Physiologic, and Electroencephalographic Effects. The Journal of Alternative and Complementary Medicine, 13(2), 109-206. doi: https://doi.org/10.1089/acm.2006.6201

Zatorre, R. J. (2015). Musical pleasure and reward: Mechanisms and dysfunction. Annals of the New York Academy of Sciences, 1337(1), 202-211. doi: https://doi. org/10.1111/nyas.12677 